\title{
A Probabilistic Geocoding System Utilising a Parcel Based Address File
}

\author{
Peter Christen $^{1, \star}$, Alan Willmore ${ }^{2}$, and Tim Churches ${ }^{2}$ \\ Department of Computer Science, Australian National University, \\ Canberra ACT 0200, Australia \\ peter.christen@anu.edu.au
}

for Epidemiology and Research, New South Wales Department of Health, Locked Mail Bag 961, North Sydney NSW 2059, Australia

\{awill, tchur\}@doh.health.nsw.gov.au

stract. It is estimated that between $80 \%$ and $90 \%$ of governmental a collections contain address information. Geocoding - the process of igning geographic coordinates to addresses - is becoming increasingly portant in application areas that involve the analysis and mining of h data. In many cases, address records are captured and/or stored a free-form or inconsistent manner. This fact complicates the task accurately matching such addresses to spatially-annotated reference a. In this paper we describe a geocoding system that is based on a nprehensive high-quality geocoded national address database. It uses earning address parser based on hidden Markov models to segment -form addresses into components, and a rule-based matching engine determine the best matches to the reference database.

\section{oduction}

ny businesses, government organisations and research projects collectve amounts of data, the techniques collectively known as data mining ntly attracted interest both from academia and industry. While there ngoing research in data mining algorithms (to extract new and unexformation from data, build predictive models, or detect outliers and ts), a large proportion of the time and effort in real world data mincts is spent understanding the data and in data preparation processes ay dominate the actual data mining activity) [14]. It is generally acthat about $20 \%$ to $30 \%$ of the time and effort in a data mining project r data understanding, and about $50 \%$ to $70 \%$ for data preparation.

paper we discuss one issue of data preparation, namely the matcher data with geocoded reference data (which comprises cleaned and sed records containing address information plus their geographical lo- 
rojects. The US Federal Geographic Data Committee estimates that ic location is a key feature in $80 \%$ to $90 \%$ of governmental data collecIn many cases, addresses are the key to spatially enable data (i.e. to data with locations). The aim of geocoding is to generate a geographion (latitude and longitude) from street address information in the user ce geocoded, the data can be used for further processing, for spatial ing [7], and it can be visualised and combined with other data using ical Information Systems (GIS).

plications of spatial data analysis and mining are widespread. In the ctor, for example, geocoded data can be used to find local clusters of nvironmental health studies often rely on GIS and geocoding software reas of potential exposure and to locate where people live in relation to as. Geocoded data can also help in the planning of new health resources, ional health care providers can be allocated close to where there is an need for such services. An overview of geographical health issues can in [1]. Geocoded client data, combined with additional demographic help businesses to better plan marketing and future expansion, and sis of historical geocoded data, for example, can show changes in their se. For national censuses, geocoding can be used to assign people or ls to small area units, for example census collection districts, which are basis of further statistical analysis.

ding can be seen as a special case of data (or record) linkage, the task of cords from one or more data sets belonging to the same entity. Most of the linkage (or matching) process is challenged by the lack of common itity identifiers, and thus becomes non-trivial $[3,8,18]$. In such cases, ble partially identifying information - like names, addresses, and dates - is used to decide if two (or more) records correspond to the same is process is computationally intensive, and linking todays large data s becomes increasingly difficult using traditional linkage techniques. lany data mining and data analysis projects, data linkage is needed ate, match, or combine information from multiple sources in order to available data and to allow more detailed analysis.

following section we discuss geocoding techniques in more detail, and 3 we present an overview of our geocoding system. The two central issues in geocoding are (1) the accurate and efficient matching of user Iresses with the address information stored in the geocoded reference (2) the efficient retrieval of the address location (latitude and lonf the matched geocoded records. In order to achieve accurate match ddresses both in the user data set and the geocoded reference data e cleaned and standardised in the same way. We cover this issue in ails in Section 3.1. Address locations can efficiently be retrieved from ded reference data by converting the traditional database tables (or 
Christen, A. Willmore, and T. Churches

\section{coding}

two basic scenarios for geocoding user data. In the first, a user wants atically geocode a (large) data set. The geocoding system should find possible match for each record in the user data set without human on. Each record needs to be attributed with the corresponding location atch status which indicates the accuracy of the match obtained (for an exact address match, or a street level match, or a locality level This scenario might become problematic if the user data is not of high and contains records with missing, incorrect, or out-of-date address on). Typographical errors are common with addresses, especially when recorded over the telephone, scanned, or manually typed from handorms. As reported in [13], a match rate of $70 \%$ successfully geocoded often considered an acceptable result.

second scenario a user interactively wants to geocode a single address all number of addresses) that may be incomplete, erroneous or unforThe system should return the location if an exact match can be found, atively a list of possible matches, together with a matching status and od rating. This geocoding of a single record should be done in (near) (i.e. less than a couple of seconds response time) and be available via user interface (for example a Web site).

Ird data (or record) linkage techniques $[3,8,18]$, where the aim is to link 1) together all records belonging to the same entity, normally classify ared record pairs into one of the three classes matches, non-matches ble matches, with the latter class containing those record pairs for which rersight, also known as clerical review, is needed to decide their final atus. This is similar to the second geocoding scenario described above, user is presented with a selection of possible matches (sorted according natching status and likelihood rating).

GIS software packages provide for street level geocoding. As a recent ws [2], substantial differences in positional error exist between addresses geocoded using street reference files (containing geographic centreline es, street numbers and names, and postcodes) and the corresponding ions. The use of point property parcel coordinates (i.e. the centres or of properties), derived from cadastral data, is expected to significantly ese positional errors. Figure 1 gives an illustrative example. Even small cies in geocoding can result in addresses being assigned to, for exament census collection districts, which can have significant implications ng small area analysis. A comprehensive property based database is able for Australia: the Geocoded National Address File (G-NAF). It is in details in the following section. 


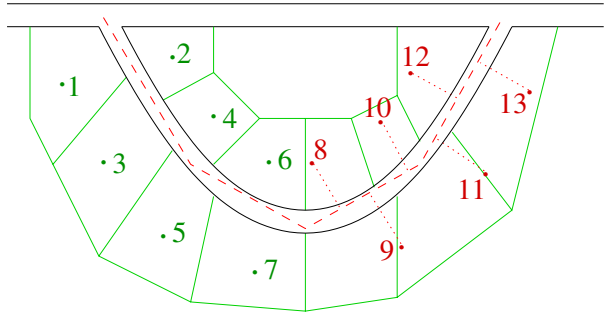

cample geocoding using property parcel centres (numbers 1 to 7 ) and street ile centreline (dashed line and numbers 8 to 13, with the dotted lines correo a global street offset)

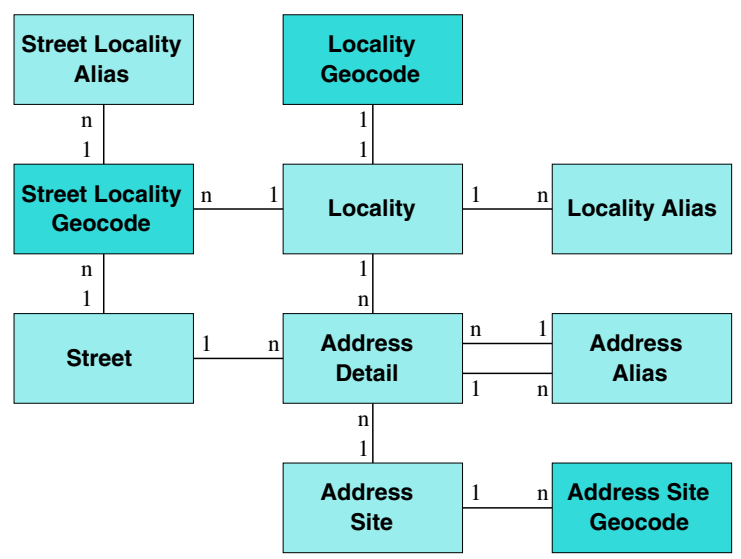

nplified G-NAF data model (10 main tables only). Links $1-n$ denote one-tolinks 1-1 denote one-to-one relationships.

lat collects data to be used for land planning, as well as property, ture or resource management. Additionally, national organisations like telecommunications, electoral rolls and statistics or census bureaus eir own data. All these data sets are collected for specific purposes, ing content and might be stored in different formats.

ed for a nation-wide, standardised and high-quality geocoded data set recognised in Australia since 1990 [13], and after years of planning, tion and development the G-NAF was first released in March 2004. ately 32 million address records from 13 organisations were used in a cleaning and integration process, resulting in a database consisting of lised tables (or files). Figure 2 shows the simplified data model of the 10 JAF tables. For our project we only used the G-NAF records covering alian state of New South Wales (NSW), containing around 4.5 million 
Christen, A. Willmore, and T. Churches

le 1. Characteristics of the 10 main G-NAF tables (NSW data only)

\begin{tabular}{|c|c|c|}
\hline ble & $\begin{array}{r}\text { Numbers of records } \\
\text { and attributes }\end{array}$ & $\begin{array}{r}\text { Keys (persistent } \\
\text { identifiers) }\end{array}$ \\
\hline _ALIAS & $333,729 / 7$ & $\begin{array}{r}\text { PRINCIPAL_PID } \\
\text { ALIAS_PID }\end{array}$ \\
\hline DETAIL & $4,585,707 / 34$ & $\begin{array}{r}\text { GNAF_PID } \\
\text { LOCALITY_PID } \\
\text { STREET_PID } \\
\text { ADDRESS_SITE_PID }\end{array}$ \\
\hline _SITE & $4,499,157 / 7$ & ADDRESS_SITE_PID \\
\hline S_SITE_GEOCODE & $3,872,217 / 15$ & ADDRESS_SITE_PID \\
\hline $\mathrm{V}$ & $5,357 /$ & LOCALITY_PID \\
\hline TY_ALIAS & $575 / 9$ & $\begin{array}{r}\text { LOCALITY_PID } \\
\text { ALIAS_PID }\end{array}$ \\
\hline Y_GEOCODE & $5,318 / 14$ & LOCALITY_PID \\
\hline & $60,586 / 6$ & STREET_PID \\
\hline _OCALITY_ALIAS & $2,940 / 10$ & $\begin{array}{r}\text { STREET_PID } \\
\text { LOCALITY_PID }\end{array}$ \\
\hline LOCALITY_GEOCODE & $130,262 / 16$ & $\begin{array}{r}\text { STREET_PID } \\
\text { LOCALITY_PID } \\
\end{array}$ \\
\hline
\end{tabular}

locations for a single address, and vice versa, and aliases are available at vels. Three geocode tables contain location (latitude and longitude) infor different levels. If an exact address match can be found, its location trieved from the ADDRESS_SITE_GEOCODE table. If there is only a match level (but not street number), then the STREET_LOCALITY_GEOCODE tarovide an overall street geocode. Finally, if no street level match can the LOCALITY_GEOCODE table contains geocode information for cities, d suburbs. The street and locality level geocode tables also contain on about the extent of streets and localities, respectively.

\section{tem Overview}

oding system presented in this paper is part of the Febrl (Freely Extennedical Record Linkage) data linkage system [3], that contains modules nd standardise data sets which can contain names, addresses and dates; and deduplicate such cleaned data. An overview of the Febrl geocoding shown in Figure 3. The geocoding process can be split into the preprothe G-NAF tables (which is described in detail in Sections 3.1 and 3.2), natching with user-supplied addresses as presented in Section 4. eprocessing step takes the G-NAF tables and uses the Febrl address 


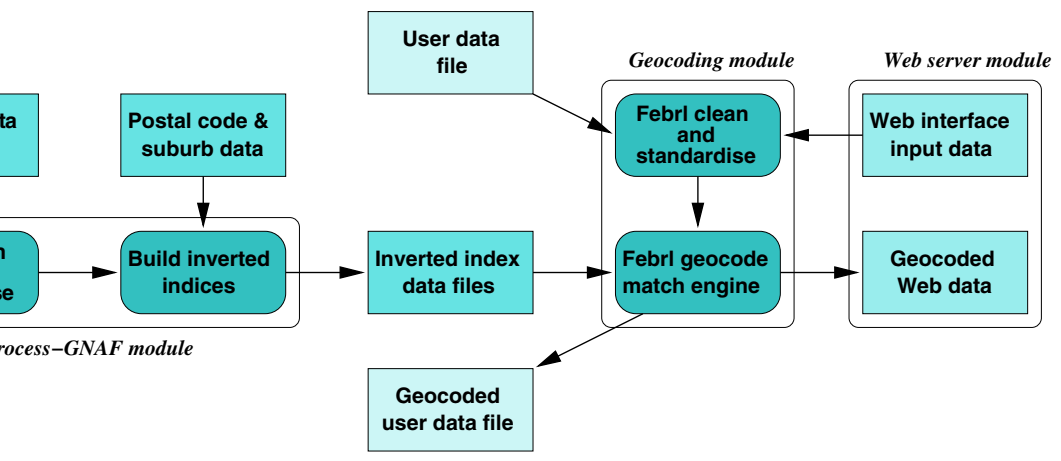

Fig. 3. Overview of the Febrl geocoding system

eady come in a highly standardised form, but the finer details, for now whitespaces within locality names are treated, make the difference successful or failed matching. The cleaned and standardised reference re then inserted into a number of inverted index data structures.

er to be able to find matches with typographical errors (like in street y names), indices based on $q$-grams (substrings of length $q$ ) are built ain address name fields. If no exact match can be found, for example n suburb name, these approximate indices will return the most similar mes available in the G-NAF.

onal data used in the preprocessing step are a postcode-suburb look-up ed on a publicly-available file made available by the Australian postal Australia Post), and which can be used to impute missing postcodes values in the G-NAF locality table; and a look-up table of adjacent and suburbs, derived from boundary information, which is used to ighbouring region look-up tables.

ocode matching engine takes as input the inverted indices and the raw , which is cleaned and standardised before geocoding is attempted. As Figure 3, the user data can either be loaded from a data file, geocoded saved into a new data file, or it can be passed as one or more addresses ocoding system via a Web interface. An extension module has been by the New South Wales Department of Health which allows geocoded a derived from such addresses to be immediately visualised as a layer based GIS (geographic information system) facility. mplete Febrl system, including the geocoding and Web server modules, ented in the object-oriented open source language Python ${ }^{1}$, which ald prototype development and testing.

babilistic Address Cleaning and Standardisation 
ding. It is commonly accepted that real world data collections contain , incomplete and incorrectly formatted information. Data cleaning and sation are important preprocessing steps for successful data linkage and 5 , and must also be carried out before including such data in a data e for further analysis [16]. Data may be recorded or captured in various, bsolete, formats and data items may be missing, out-of-date, or contain ne cleaning and standardisation of addresses is especially important for age and geocoding so that accurate matching results can be achieved. ain task in the cleaning and standardisation of addresses is the convere raw input data into well defined, consistent forms and the resolution of ncies in the manner in which address values are represented or encoded. d data cleaning and standardisation, as currently used by many comrstems, can be cumbersome to set up and maintain, and often needs adfor new data sets. We have recently developed (and implemented within obabilistic techniques [4] based on hidden Markov models (HMMs) [15] iieved better address standardisation accuracy and which seem to be set up and maintain than rule-based address processing software.

$\mathrm{M}$ is a probabilistic finite-state machine consisting of a set of observation symbols, a finite set of discrete, hidden (unobserved) states, a matrix of probabilities between those hidden states, and a matrix of probabilities ch each hidden state emits an observation symbol [15] (this emission also sometimes referred to as an observation matrix). In our case, the ates of the HMM correspond to the output fields of the standardised

ig data are representative samples of the input records which have been into sequences of observation symbols as described below (steps 1 and hen tagged with the hidden state which the (human) trainer thought likely to have been responsible for emitting each observation symbol. likelihood estimates (MLEs) are derived for the HMM transition and probability matrices by accumulating frequency counts for each type ransition and observation symbol from the training records [4]. The pear to be quite robust with respect to the training set used and quite ith respect to the data sources with which they can be used. As a is quite feasible to add specific training records which are archetypes of ddress patterns, without compromising the performance of the HMMs ypical source records.

ebrl approach to address cleaning and standardisation consists of the three steps.

iser input addresses are cleaned. This involves converting all letters to -case, removing certain characters (such as punctuation), and convertrious sub-strings into their canonical form, for example ' $c /-$ ', 'c/o' and 
I approach produced equal or better standardisation accuracies than y-used rule-based system AutoStan [10], which uses a re-entrant, regular n-like pattern matching approach.

\section{ocessing the G-NAF Files}

$\mathrm{g}$ the G-NAF tables consists of two steps, the first being the cleaning lardisation as described above, and the second step being the buildzerted indices. An inverted index is a keyed hash-table in which the he values from the cleaned G-NAF tables, and the entries in the hashsets with the corresponding PIDs (persistent identifiers, see Table 1) lues. For example, assume there are four records in the LOCALITY tathe following content (the first line is a header-line with the attribute

$\begin{array}{llll}\text { ocality_pid, } & \text { locality_name, } & \text { state_abbrev, } & \text { postcode } \\ \text { 0310919, } & \text { sydney, } & \text { nsw, } & 2000 \\ \text { 0709845, } & \text { north_sydney, } & \text { nsw, } & 2059 \\ 0309156, & \text { north_sydney, } & \text { nsw, } & 2060 \\ 1560124, & \text { the_rocks, } & \text { nsw, } & 2000\end{array}$

ted indices for the three attributes locality_name, state_abbrev and then are (curly brackets denote keyed hash-tables and round brackets ts):

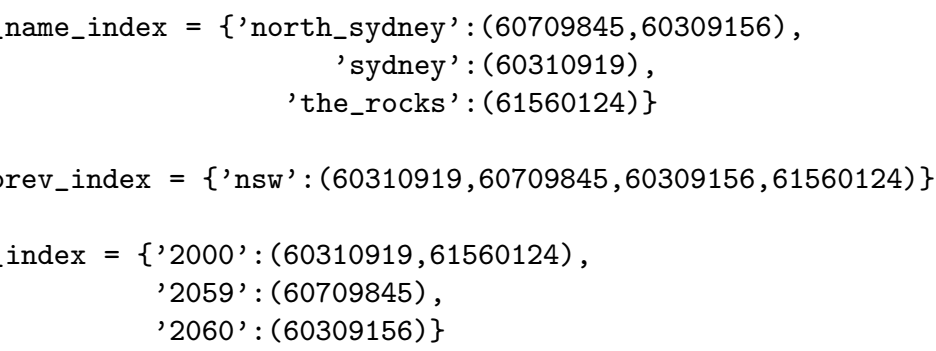

hing engine then finds intersections of the inverted index sets for the a given record. For example, a postcode value '2000' would result f PIDs $(60310919,61560124)$ being returned, and when intersected PIDs returned for locality name value 'the_rocks' would result in the set (61560124), which corresponds to the original record. The geocation of this PID can then be looked-up in the corresponding G-NAF ndex. Table 2 shows the 23 attributes for which inverted indices are 
Table 2. G-NAF attributes used for geocode matching

\begin{tabular}{ll}
\hline ble & Attributes used \\
\hline S_DETAIL & flat_number_prefix, flat_number, flat_number_suffix, \\
& flat_type, level_number, level_type, building_name, \\
& location_description, number_first_prefix, \\
& number_first, number_first_suffix, number_last_prefix, \\
& number_last, number_last_suffix, lot_number_prefix, \\
& lot_number, lot_number_suffix \\
'Y_ALIAS & locality_name, postcode, state_abbrev \\
& locality_name, postcode, state_abbrev \\
LOCALITY_ALIAS & street_name, street_type, street_suffix \\
& street_name, street_type, street_suffix \\
\hline
\end{tabular}

and then calculating a similarity value (sometimes called the Dice co5])

$$
\text { sim_value }=2 \times\left(\frac{\operatorname{qgram}(x) \cap \operatorname{qgram}(y)}{|\operatorname{qgam}(x)|+|\operatorname{qram}(y)|}\right)
$$

$\operatorname{arm}(s)$ is a function which reduces a string $s$ to its set of $q$-grams. This measure can have values between 0.0 (no $q$-grams in common) and grams in common, i.e. the strings are the same). A ranked list of all ate matches that have a similarity value above a user defined threshold used in the matching process for a given user record.

\section{ditional Data Files}

l information is used in the Febrl geocoding system during the preproep to verify and correct (if possible) postcode and locality name values, e matching engine to enable searching for matches in neighbouring restcodes and suburbs) if no exact match can be found.

lia Post publishes a look-up table containing postcode and suburb in$2^{2}$, which is used when processing the G-NAF locality tables to verify ct wrong or missing postcodes and suburb names. For example, if a is missing in a record, the Australia Post look-up table can be used to fficial postcode(s) for the suburb in this record, and if this is a unique it can be safely imputed into the record. Similarly, missing suburb $\mathrm{n}$ be imputed if they correspond to a unique postcode.

look-up tables are used to find neighbouring regions for postcodes and i.e. for a given region these tables contain all its neighbours. These tables were derived from region boundary data using well-known GIS s. Look-up tables of both direct and indirect neighbours (i.e. neigh- 
Christen, A. Willmore, and T. Churches

ce has shown that people often record different postcode or suburb valeighbouring postcode or suburb has a higher perceived social status, or e close to the border of such regions.

\section{code Matching Engine}

ode matching engine in Febrl is based on the G-NAF inverted hash nd takes a deterministic but adaptive approach to find an exact match atively one or more approximate matches. Its input is one or many nd standardised user record(s).

atching engine tries to find an exact match first, but if none can be extends its search to neighbouring postcode and suburb regions. First ghbouring regions (level 1) are searched, then direct and indirect neighegions (level 2), until either an exact match or a set of approximate can been found. In the latter case, either an average location over all matches is returned, or a ranked (according to a matching weight) list e matches. The following steps explain in more detail (but still at a al level) how the matching engine works.

the set of address level matches (using street number and suffix) and et of street level matches (using street name and type). Street names st searched using exact string comparison, and if no street name match e found approximate string matching will be applied (using a $q$-gram ), allowing to find matches with typographical errors.

e neighbouring search level to 0 (no neighbouring regions are searched). the locality level matches (using locality name, qualifier and postcode) ding to the current value of the neighbouring search level. Locality s are first searched using exact string comparison, and if no locality match can be found approximate string matching will be applied (using cam index), allowing to find matches with typographical errors. Postare only used in the matching process if they result in the same matches locality names (i.e. if they contribute to these matches). If postcodes in different matches, they are not used in the final match (this is bepostcodes are not fixed locality regions, but can be - and often are ed by Australia Post for the purposes of expediting delivery of mail). for matches between street level and locality level, and if they exist e the set of street level matches using the locality level matches, and flag street_locality_match to true.

: for matches between address level and locality level, and if they exist e the set of address level matches using the locality level matches, and flag address_locality_match to true.

for matches between address level and street level, and if they exist 
e values of the three matching flags. A match has been found if all match flags are true, or if there was no address level match and the locality_match flag is true, or if there was no street level match and ddress_locality_match flag is true, or if there was no locality level match he address_street_match flag is true. If no match has been found then ase the neighbouring search level -that is, widen the search to adjacent as - (up to a maximum of 2) and go back to step 3.

eral matches have been found, try to refine them using unit, flat and ng (or property) information (if such information is available in the nput record).

ine the match(es) from the different levels (basically get their set interns), and set the final match level (to either address, street or locality). eve the coordinates of the match(es) from the corresponding G-NAF de index (address site, street/locality or locality). If one match has been retrieve it's coordinates and return them together with the G-NAF (persistent identifier). If several matches were found check if they are a a small area (defined by the user). If so, average their coordinates and a the averaged location together with the list of G-NAF PIDs. If the aces between the matches were large, return a 'Many match' (without inates, only a list of G-NAF PIDs).

me cases a match at a certain level has been found, but no record is ble in the corresponding G-NAF geocode index, or a record is availout does not contain coordinates. In this case go to the next higher a level (e.g. from address to street, or from street to locality) and try rieve the corresponding coordinates (back to step 10).

match was found return a 'No match' match status.

$\mathrm{g}$ of multiple addresses is an iterative process where each record is first nd standardised, then geocoded and written into an output data set dinates and a match status added to each record.

\section{erimental Results}

run experiments with geocoding various administrative health data address hidden Markov model (HMM) was trained using 1,300 trainds, around half of them randomly selected from the NSW Midwives lection [11] (MDC) containing data from 1990 to 2000, the other half $\mathrm{m}$ a NSW Land and Property Information (LPI) data set from 2002. sual training records (like rural addresses or addresses with property tion information) were manually added to increase the standardisation for such addresses.

3 presents the results of geocoding 10,000 randomly selected LPI free- 
Christen, A. Willmore, and T. Churches

Matching results for geocoding 10,000 free-form LPI address records, with d without $(q=0)$ approximate indices

\begin{tabular}{lrrrr}
\hline & Number of records & \multicolumn{2}{c}{ Percentage } \\
ch status & $q=2$ & $q=0$ & $q=2$ & $q=0$ \\
\hline ct address level match & 7,525 & 7,497 & $75.27 \%$ & $74.97 \%$ \\
rage address level match & 303 & 303 & $3.03 \%$ & $3.03 \%$ \\
y address level match & 178 & 178 & $1.78 \%$ & $1.78 \%$ \\
ct street level match & 1,160 & 1,131 & $11.60 \%$ & $11.31 \%$ \\
rage street level match & 17 & 17 & $0.17 \%$ & $0.17 \%$ \\
y street level match & 65 & 62 & $0.65 \%$ & $0.62 \%$ \\
ct locality level match & 653 & 680 & $6.53 \%$ & $6.80 \%$ \\
rage locality level match & 1 & 1 & $0.01 \%$ & $0.01 \%$ \\
y locality level match & 98 & 118 & $0.98 \%$ & $1.18 \%$ \\
\hline
\end{tabular}

le 4. Comparative matching results for geocoding $873 \mathrm{MDC}$ addresses

\begin{tabular}{lrrrr}
\hline \multirow{2}{*}{ status } & \multicolumn{2}{c}{ Number of records } & \multicolumn{2}{c}{ Percentage } \\
& Febrl & Commercial & Febrl & Commercial \\
\hline address level match & 746 & 789 & $85.45 \%$ & $90.38 \%$ \\
e address level match & 23 & - & $2.63 \%$ & - \\
address level match & 13 & - & $1.49 \%$ & - \\
street level match & 48 & 37 & $5.50 \%$ & $4.24 \%$ \\
street level match & 3 & - & $0.34 \%$ & - \\
locality level match & 35 & 46 & $4.01 \%$ & $5.26 \%$ \\
locality level match & 4 & - & $0.46 \%$ & - \\
tch & 1 & 1 & $0.11 \%$ & $0.11 \%$ \\
\hline
\end{tabular}

. Comparative matching results for geocoding 607 nursing home addresses

\begin{tabular}{lrrrr}
\hline & \multicolumn{2}{c}{ Number of records } & \multicolumn{2}{c}{ Percentage } \\
status & Febrl & Commercial & Febrl & Commercial \\
\hline address level match & 265 & 384 & $43.66 \%$ & $63.26 \%$ \\
e address level match & 30 & - & $4.94 \%$ & - \\
dddress level match & 21 & - & $3.46 \%$ & - \\
treet level match & 240 & 202 & $39.54 \%$ & $33.28 \%$ \\
e street level match & 1 & - & $0.16 \%$ & - \\
treet level match & 4 & - & $0.66 \%$ & - \\
ocality level match & 38 & - & $6.26 \%$ & -
\end{tabular}


tivated, the approximate indices allowed for up to two typographical . single character insertions, deletions, or substitutions) in street and ames. As the results show, a total of $93.38 \%$ (with approximate indices) $\%$ (without) exact matches were found at different levels. Using approxices resulted in only a slightly improved match quality, but geocoding h longer. Without approximate indices, the average geocoding time ss was 67 milliseconds; using approximate indices it increased to 269 ids per address (around four times slower).

le 4 the results of a geocoding experiment on 873 randomly selected ad$1 \%$ sample) from the MDC using the Febrl geocoder are compared to cial (street centreline based) geocoding system (which only returns exnes). The 'no match' address in both cases was for an overseas address. nows comparative results on a NSW nursing home data sets containing esses. For this data set the commercial system achieves a significantly ocoding quality. This is because nursing homes are often located at ersections and have corner addresses. Such addresses are currently not oroperly in our geocoding system, as they correspond to two G-NAF letail records (with the same address site), and cannot be separated by our address standardiser.

lso that the comparison results in Tables 4 and 5 only show the match it not the relative accuracy of the coordinates returned. We are planning tore detailed analysis on this issue, comparable to the study presented

\section{clusions and Future Work}

per we have described a geocoding system based on a geocoded national le. We are currently evaluating and improving this system using raw addresses taken from various administrative health related data sets. lso planning to compare the accuracy of our geocoding system with sed geocoders, and similar to [2] we expect to obtain more accurate ur geocoding system has been published as part of the Febrl data rstem [3] under an open source software license. Readers should note ugh our geocoding engine is available for free, the G-NAF reference files uses are not freely available, and must be licensed through PSMA [13] tner organisations.

efforts will be directed towards the refinement of the geocode matche to achieve more accurate matching results, as well as improving the nce of the matching engine (i.e. reducing the time needed to match an Three other areas of future work include:

Febrl standardisation routines currently return fields (or attributes) which fferent from the ones available in the G-NAF. This makes it necessary to 
Christen, A. Willmore, and T. Churches

ntly both the G-NAF preprocessing and indexing, as well as the geocode ing engine work in a sequential fashion only. Due to the large data files ed, parallel processing becomes desirable. In the preprocessing step, the Gtables can be processed independently or in a block-wise fashion, distributed number of processors, with only the final inverted indices needing to be d. Geocoding of a large user data file can easily be done in parallel as the ng, standardisation and matching of each record is completely independent all others. An additional advantage of parallelisation is the increased amount in memory available on many parallel platforms. We are planning to explore arallelisation techniques and implement them into the Febrl system to allow geocoding of larger data sets. Additional performance improvements can be ed by profiling and re-implementing the core computational routines as $\mathrm{C}$ age routines called from the Python code (something which Python makes o do).

$\mathrm{r}$ addresses are stored in the G-NAF as two separate addresses with the same ss site PID, but when processed in a user data set this becomes problematic, standardisation process is currently not capable of separating the compoof a corner address. We aim to work on this issue and add it to a future n of our geocoding system.

ding uses identifying information (i.e. addresses) which raises privacy and entiality issues. Organisations that collect sensitive data (e.g. disease reg) cannot send their data to a third-party geocoding service as this may result loss of privacy for individuals involved. It is desirable to develop methods allow for privacy-preserving geocoding. We aim to develop such methods on techniques recently described for blindfolded data linkage $[5,9,12]$.

\section{vledgements}

is funded by the NSW Department of Health, Centre for Epidemiology arch. The authors would like to thank David Horgan (student at the y of Queensland) who worked on a first version of this system while summer student at the ANU. The authors also wish to thank the partment of Lands for facilitating access to the G-NAF for research opment purposes.

\section{aces}

s, M.N.K.: Towards evidence-based, GIS-driven national spatial health ation infrastructure and surveillance services in the United Kingdom. ational Journal of Health Geographics 2004, 3:1. Available online at: //www.ij-healthgeographics.com/content/3/1/1

M.R. and Talbot, T.O.: Positional error in automated geocoding of residenAdresses. International Journal of Health Geographics 2003, 2:10. Available 
hes, T., Christen, P., Lim, K. and Zhu, J.X.: Preparation of name and addata for record linkage using hidden Markov models. BioMed Central Medformatics and Decision Making 2002, 2:9, Dec. 2002. Available online at: //www . biomedcentral . com/1472-6947/2/9/

hes, T. and Christen, P.: Some methods for blindfolded record linkage. d Central Medical Informatics and Decision Making 2004, 4:9, June 2004. ble online at: http://www.biomedcentral .com/1472-6947/4/9/

r, C.: The CRISP-DM Model: The new blueprint for data mining. Journal a Warehousing, vol. 5, no. 4, pp. 13-22, Fall 2000.

M., Kriegel, H.-P. and Sander, J.: Spatial Data Mining: A Database Ap, Fifth Symposium on Large Spatial Databases (SSD'97). Springer LNCS pp. 48-66, 1997.

, I. and Sunter, A.: A Theory for Record Linkage. Journal of the American tical Society, 1969.

.: Development of a Blind Geocoding System. Honours thesis, Department nputer Science, Australian National University, Canberra, November 2004. 'tan and AutoMatch, User's Manuals, MatchWare Technologies, Kennebunk, 1998.

for Epidemiology and Research, NSW Department of Health. New South Mothers and Babies 2001. NSW Public Health Bull 2002; 13(S-4).

fe, C.M., Yung, M., Gu, L. and Baxter, R.: Privacy-Preserving Data Linkcotocols. Proceedings of the Workshop on Privacy in the Electronic Society 'S'04). Washington, DC, October 2004.

D.L.: A geocoded National Address File for Australia: The G-NAF What, Who and When? PSMA Australia Limited, Griffith, ACT, Australia, 2003. ble online at: http://www.g-naf .com.au/

D.: Data Preparation for Data Mining. Morgan Kaufmann Publishers, Inc.,

er, L.R.: A Tutorial on Hidden Markov Models and Selected Applications in n Recognition. Proceedings of the IEEE, vol. 77, no. 2, Feb. 1989.

, E. and Do, H.H.: Data Cleaning: Problems and Current Approaches. IEEE Engineering Bulletin, 2000.

deral Geographic Data Committee. Homeland Security and Geographic Intion Systems - How GIS and mapping technology can save lives and protect ty in post-September 11th America. Public Health GIS News and Informa1o. 52, pp. 21-23, May 2003.

er, W.E.: The State of Record Linkage and Current Research Problems. 03, US Bureau of the Census, 1999. 